\title{
Direct Antiglobulin Test-Negative Autoimmune Hemolytic Anemia
}

\author{
Tsuyoshi Takahashi \\ Department of Hematology, Nerima Hikarigaoka Hospital, Tokyo, Japan
}

Warm autoimmune hemolytic anemia (AIHA) is a rare type of anemia caused by the reaction of auto-antibodies with red blood cell (RBC) surface antigens at body temperature, leading to the hemolysis of RBCs [1]. The direct antiglobulin test (DAT) (also known as the direct Coombs test) was first described by Coombs, Mourant, and Race in 1945 [2] and is still an essential assay for the diagnosis of AIHA. The DAT can detect RBC-bound IgG and complement (C3), which opsonize RBCs. However, $5-10 \%$ of patients with AIHA are DAT negative [3]. The principal reasons for DAT-negative AIHA are: (a) RBCbound IgG being below the threshold of detection by standard methods; (b) low affinity of IgG; and (c) RBCbound IgA or rarely IgM [4]. To achieve greater sensitivity, alternative DAT methods have been developed, such as the micro-column (gel), solid-phase, polybrene, and flow cytometry methods, as well as enzyme-linked antiglobulin, immunoradiometric, and mitogen-stimulated tests [4]. Moreover, because washing RBCs with room temperature saline causes the low-affinity antibodies to be eluted from RBCs, washing RBCs with cold saline or with low ionic strength saline (LISS) may be effective in detecting low-affinity antibodies.

Because the prevalence of DAT-negative AIHA is low, the relative proportions of each of the aforementioned

\section{KARGER}

() 2018 S. Karger AG, Basel

E-Mail karger@karger.com

www.karger.com/aha reasons for DAT-negative AIHA have not been clarified. Moreover, a diagnostic algorithm for DAT-negative AIHA has not yet been established.

In this issue of the journal, Kamesaki and Kajii [5] report a detailed laboratory analysis of patients with DATnegative AIHA and propose a classification of DAT-negative AIHA using a combination of several DAT methods. They studied patients with undiagnosed hemolytic anemia who showed negative results using the routine tube method of DAT (TM-DAT), who were further investigated with anti-IgA and anti-IgM sera and the column agglutination method of DAT (CM-DAT), and with immunoradiometric quantitation of RBC-bound IgG. According to the results, they proposed a classification of DAT-negative AIHA into 5 groups as follows: (1) positive CM-DAT after washing RBCs with phosphate-buffered saline (PBS) and $\mathrm{RBC}$-bound IgG in the immunoradiometric assay over the cutoff value (the cutoff value of RBC-bound IgG is $78.5 \mathrm{IgG}$ molecules/RBC [6]); (2) negative CM-DAT and $\mathrm{RBC}$-bound IgG in the immunoradiometric assay over the cutoff value; (3) negative CM-DAT after washing RBCs with PBS, but positive TM- or CM-DAT after washing RBCs with LISS and RBC-bound IgG in the immunoradiometric assay under the cutoff value; (4) negative CMDAT after washing RBCs with PBS, but positive TM- or 
CM-DAT after washing RBCs with LISS and RBC-bound IgG in the immunoradiometric assay over the cutoff value; and (5) positive TM-DAT with anti-IgA or anti-IgM sera. The estimated relative fractions of patients with DATnegative AIHA resulting from RBC-bound IgA or IgM, low-affinity IgG autoantibodies, and RBC-bound IgG autoantibodies under the threshold level of positive DAT were approximately 5,15 , and $80 \%$, respectively.

The study by Kamesaki and Kajii [5] indicated that most cases of DAT-negative AIHA were due to RBCbound IgG being below the threshold of detection by standard methods, followed by cases of low-affinity IgG and $\mathrm{RBC}$-bound IgA or IgM. When AIHA patients are TM-DAT negative, the next step may be to evaluate RBC- bound IgG by an immunoradiometric method, then with low-affinity IgG autoantibodies or with anti-IgA or IgM sera. However, there are many other DAT methods, and it is a challenge to choose which combination of methods to use. Moreover, we should keep in mind that these tests for DAT-negative AIHA have a low predictive value, and thus the results must be interpreted based on the clinical status of the patients.

Nevertheless, Kamesaki and Kajii's [5] work in this issue casts a new light on DAT-negative AIHA. This classification of DAT-negative AIHA can reduce misdiagnosis rates and may be useful in predicting the prognosis of each DAT-negative AIHA type. Further development in this field will be expected.

\section{References}

1 Kalfa TA: Warm antibody autoimmune hemolytic anemia. Hematology Am Soc Hematol Educ Program 2016;2016:690-697.

2 Coombs RR, Mourant AE, Race RR: A new test for the detection of weak and incomplete Rh agglutinins. Br J Exp Pathol 1945;26:255266.

3 Garratty G: Immune hemolytic anemia associated with negative routine serology. Semin Hematol 2005;42:156-164

4 Parker V, Tormey CA: The direct antiglobulin test: indications, interpretation, and pit- falls. Arch Pathol Lab Med 2017;141:305-310.

5 Kamesaki T, Kajii E: A comprehensive diagnostic algorithm for direct antiglobulin testnegative autoimmune hemolytic anemia reveals the relative ratio of three mechanisms in a single laboratory. Acta Haematol 2018, DOI: $10.1159 / 000488753$.

6 Kamesaki T, Oyamada T, Omine M, Ozawa K, Kajii E: Cut-off value of red-blood-cellbound IgG for the diagnosis of Coombs-negative autoimmune hemolytic anemia. Am J Hematol 2009;84:98-101. 